\title{
COUNCIL AND BOARD OF TRUSTEES. 1974 and 1975
}

Except for the Members-at-Large of the Council, the month and year of the first term and the end of the present term are given. For Members-at-Large of the Council, the last year of the present term is listed.

\section{COUNCIL}

President: Lipman Bers 1/75-12/76

Saunders Mac Lane 1/73-12/74

Past President: Saunders Mac Lane 1/75-12/75

Vice Presidents:

Raoul H. Bott 1/74-12/75

Irving Kaplansky 1/74-12/75

John W. Milnor 1/75-12/76

Edwin E. Moise 1/73-12/74
Secretary: Everett Pitcher 1/67-12/76

Associate Secretaries:

Paul Bateman 1/67-12/75

Walter Gottschalk 1/71-12/76

Orville G. Harrold, Jr. 1/65-12/76

Kenneth A. Ross 1/71-12/75

Treasurer: Franklin Peterson 1/73-12/76

Associate Treasurer:

Murray H. Protter 1/73-12/76

\section{Publications and Communications Committees}

Bulletin Editorial Committee

Paul R. Halmos 1/74-12/76

John L. Kelley 1/73-12/75

Hans F. Weinberger 1/72-12/77

Proceedings Editorial Committee

Fred Brauer 1/71-9/74

Glen E. Bredon 1/72-12/75

W. Wistar Comfort 1/72-12/75

Chandler Davis 9/74-12/75

Jacob Feldman 1/72-12/76

(on leave 8/74-1975)

Robert M. Fossum 1/74-12/77

Richard R. Goldberg 1/72-12/75

Barbara L. Osofsky 1/74-12/77

Richard K. Miller 9/74-12/78

Transactions and Memoirs Editorial Committee Philip T. Church 1/74-12/77

Alexandra Ionescu-Tulcea 10/74-12/77

Harry Kesten 1/71-12/74

Alistair H. Lachlan 1/72-12/75

Dock S. Rim 1/71-12/74

Stephen S. Shatz 1/75-12/78

Shlomo Sternberg 1/70-9/74

Daniel W. Stroock 1/75-12/78

François Treves $1 / 72-12 / 75$
Representatives on American Journal of Mathematics

Hyman Bass 1/71-12/76

I. M. Singer 1/72-12/77

Mathematical Reviews Editorial Committee Robert G. Bartle 1/74-12/76

Frederick W. Gehring 1/72-12/74

D. J. Lewis $1 / 75-12 / 77$

Jacob T. Schwartz 1/73-12/75

Mathematical Surveys Editorial Committee Robert G. Bartle 1/72-12/77

Edgar H. Brown, Jr. 1/68-12/76

Paul R. Halmos 1/73-12/75

Mathematics of Computation Editorial Committee

James H. Bramble 1/73-12/77

Walter Gautschi 1/75-12/77

Alston S. Householder 1/73-12/76

Eugene Isaacson 1/66-12/74

John W. Wrench, Jr. 1/73-12/75

Colloquium Editorial Committee

Alberto P. Calderón 1/71-12/76

S. S. Chern 1/72-12/77

Samuel Eilenberg 1/73-12/75

Committee to Monitor Problems in Communication Leonard Gillman 1/75-12/75 Allen Shields 1/74-12/74 


\section{Members-at-Large}

All terms are for 3 years and expire on December 31 of the given year.

1974

William Browder

P. S. Mostert

Robert T. Seeley

Dorothy Maharam Stone

Olga Taussky Todd

1976
1975

Anatole Beck

Michael Golomb

Mary W. Gray

Arthur P. Mattuck

Cathleen S. Morawetz

1977

Charles W. Curtis

Herbert B. Keller

Robion C. Kirby

Lee Lorch

Jane Cronin Scanlon

\section{David Gale}

Judy Green

Phillip A. Griffiths

Karl K. Norton

J. Ernest Wilkins, Jr.

\section{BOARD OF TRUSTEES}

Paul T. Bateman 1/71-12/75

Lipman Bers (ex officio) 1/75-12/76

Calvin C. Moore 1/71-12/79

Richard S. Palais 1/72-12/76
Franklin P. Peterson (ex officio) 1/73-12/76

Murray H. Protter (ex officio) 1/73-12/76

Alex Rosenberg 1/74-12/78

Abraham H. Taub 1/73-12/77 